\title{
A perspectiva dos pais sobre a reabilitação fisioterapêutica de crianças com deficiência múltipla
}

\section{The parent's perspective on the physiotherapy rehabilitation of children with multiple disabilities}

(DEdilene Araújo Pamplona1, (DRobson da Fonseca Neves², (DAndressa Medeiros Araújo ${ }^{1}$, (DGeraldo Eduardo Guedes de Brito², (DFranklin Delano Soares Forte², (D) Kátia Suely Queiroz Silva Ribeiro²

\section{RESUMO}

A deficiência múltipla (DM) é caracterizada pela presença de duas ou mais deficiências que são capazes de influenciar o desenvolvimento e a funcionalidade dos indivíduos que a possui. A reabilitação nestes casos passa invariavelmente pela atuação fisioterapêutica que pode proporcionar ganhos funcionais e sociais para a crianças que sofrem com a DM e para suas famílias. Objetivo: Analisar como a literatura científica aborda a percepção dos pais em relação ao tratamento fisioterapêutico de crianças com deficiência múltipla. Método: Foi realizada uma metassíntese qualitativa por meio de uma busca sistemática de estudos qualitativos publicados entre 2013 e 2018 nas bases de dados Scopus e PsycINFO. A análise dos dados consistiu em três etapas propostas: sistematização dos conceitos de primeira ordem, que envolvem a identificação dos conceitos encontrados nos artigos originais; a interpretação de segunda ordem, processo de comparação e interpretação de conceitos que se repetem e, por fim, a síntese interpretativa, que são uma reinterpretação dos conceitos de segunda ordem. Resultados: Foram incluídos oito estudos e deles emergiram cinco conceitos de segunda ordem que serviram de base para a elaboração de duas sínteses interpretativas: Participação dos pais no processo de reabilitação e Informação e comunicação entre pais e fisioterapeutas na percepção das melhoras físicas obtidas com o tratamento. Conclusão: Os estudos apontaram que os pais percebem a eficácia do tratamento fisioterapêutico, no entanto, foram identificadas algumas barreiras que dificultam a realização de uma intervenção adequada.

Palavras-chave: Crianças com Deficiência, Modalidades de Fisioterapia, Reabilitação, Pesquisa Qualitativa

${ }^{1}$ Fisioterapeuta, Universidade Federal da Paraíba - UFPB

2Professor, Departamento de Fisioterapia, Universidade Federal da Paraíba - UFPB

${ }^{3}$ Professor, Departamento de Clínica Odontologia Social, Universidade Federal da Paraíba - UFPB

\section{Correspondência}

Edilene Araújo Pamplona

E-mail: rebeca.boltes@hc.fm.usp.br

Submetido: 11 Março 2020

Aceito: 27 Abril 2020

\section{Como Citar}

Pamplona EA, Neves RF, Araújo AM, Brito GEG Forte FDS, Ribeiro KSOS. A perspectiva dos pais sobre a reabilitação fisioterapêutica de crianças com deficiência múltipla. Acta Fisiatr. Acta Fisiatr. 2019;26(4):220-229.

DOI: 10.11606/issn.2317-0190.v26i4a167642

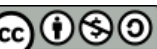

\section{ABSTRACT}

Multiple disability (MD) is characterized by the presence of two or more deficiencies that are capable of influencing the development and functionality of the individuals who have it. Rehabilitation in these cases invariably involves physical therapy that can provide functional and social gains for children suffering from MD and their families. Objective: Analyze how the scientific literature addresses the perception of parents regarding the physical therapy treatment of children with multiple disability. Methods: A qualitative metasynthesis was performed through a systematic earch of qualitative studies published between 2013 and 2018 in the Scopus and PsycINFO databases. The data analysis consisted of three stages proposed: systematization of the first order concepts, which involve the identification of the concepts found in the original articles; the second order interpretation, process of comparison and interpretation of concepts that are repeated and, finally, the interpretative synthesis, which are a reinterpretation of second order concepts. Results: Eight studies were included and from them emerged five second order concepts that served as the basis for the elaboration of two interpretative syntheses: Parent's participation in the process of rehabilitation and Information and communication between parents and physiotherapists in the perception of physical improvements obtained with the treatment. Concluision: The studies pointed out that parents perceive the efficacy of physiotherapeutic treatment, however, some barriers have been identified that make it difficult to carry out an adequate intervention.

Keywords: Disabled Children, Physical Therapy Modalities, Rehabilitation, Qualitative Research 


\section{INTRODUÇÃO}

O surto de microcefalia ocorrido no Brasil no final do ano de 2015 colocou em relevo a necessidade de compreender melhor o fenômeno e buscar estratégias para o enfrentamento do problema. ${ }^{2-4}$ Mais que isso, colocou em evidência o quanto precisamos refletir mais sobre as abordagens de reabilitação empregadas para crianças que sofrem com mais de uma deficiência, comumente chamadas de crianças com deficiências múltiplas (DM), definida como a presença de duas ou mais deficiências, sejam elas de caráter mental, físico, sensorial ou outros, capazes de influenciar o desenvolvimento e a funcionalidade dos indivíduos que a possui. ${ }^{5}$

Neste cenário, o tratamento fisioterapêutico tanto no Brasil como em todo o mundo tem papel relevante, uma vez que pode oferecer intervenções capazes de minimizar as deficiências provenientes da lesão do Sistema Nervoso Central (SNC) e de outros sistemas, favorecendo a independência funcional e consequentemente melhorando a qualidade de vida dessas crianças. ${ }^{6}$

$O$ destaque dado ao tratamento fisioterapêutico aqui não diminui o papel dos demais entes que compõem a equipe de reabilitação nos casos de DM, os quais são, aqui, reconhecidos como essenciais.

Todavia, a necessidade de conhecer mais especificamente sobre a fisioterapia e sobre o fisioterapeuta neste processo, levou-nos a destacá-los da equipe multidisciplinar, tão comum nas abordagem das DM, para que pudéssemos enxergar melhor o fenômeno da percepção que os pais têm sobre os elementos que compõem o tratamento fisioterapêutico nesses casos.

Advoga-se que, mais que a qualidade do que é ofertado em temos de tratamento fisioterapêutico, a participação dos pais neste processo é fundamental, pois pode ser um agente de manutenção e replicação de condutas nos ambientes externos aos sets terapêuticos.

Contudo, pouco se sabe sobre como se organiza a oferta e o processo de trabalho da reabilitação fisioterapêutica de crianças que sofrem de mais de uma deficiência e menos ainda sobre como os pais percebem o tratamento de fisioterapia ofertado. Por isso, considerouse a necessidade de conduzir um estudo de revisão de literatura, no qual o elemento central fossem os aspectos subjetivos da percepção dos pais sobre o tratamento de fisioterapia dispensado às crianças com DM.

Sabe-se que a atuação fisioterapêutica é capaz de potencializar na criança o desenvolvimento de habilidades motoras, a interação com o ambiente e a participação na vida familiar e comunitária. ${ }^{6}$ No entanto, é fundamental que os pais e cuidadores também estejam envolvidos e participem desse processo para que possam perceber os benefícios desse tipo de assistência e se engajem no processo de reabilitação com sua participação.

Para isso, urge também que os profissionais sejam capazes de desenvolver relações de proximidade com os pais e forneçam orientações e outros recursos que os auxiliem a compreender melhor para atuar nas necessidades especiais dos seus filhos. ${ }^{7}$

A partir dessa compreensão, acredita-se que os pais podem se sentir mais motivados a participar de forma mais concreta no tratamento e os profissionais podem obter maior eficácia no alcance dos seus objetivos terapêuticos. Assim, julga-se importante conhecer o que os pais pensam sobre os aspectos que envolvem esse tratamento.

Por isso, questiona-se: como a literatura científica de cunho qualitativo tem relatado a percepção dos pais sobre a reabilitação fisioterapêutica de filhos com DM?

\section{OBJETIVO}

Partindo desses pressupostos, o objetivo do estudo foi analisar como a literatura científica aborda a percepção que os pais têm em relação aos aspectos que envolvem o tratamento fisioterapêutico de seus filhos com DM.

\section{MÉTODO}

A metodologia do presente estudo segue a abordagem da metassíntese qualitativa, que tem como objetivo produzir uma síntese interpretativa dos dados a partir de uma integração de resultados de estudos qualitativos. Desse modo, pode ser produzida uma nova interpretação dos resultados que não é encontrada nos estudos primários analisados. ${ }^{8}$

A metassíntese qualitativa favorece uma maior profundidade de análise dos estudos qualitativos. ${ }^{9}$ É realizada uma interpretação por parte do pesquisador sobre os resultados obtidos nos estudos primários, elaborados pelos autores das pesquisas e estes estudos são selecionados de acordo com o interesse de investigação do pesquisador sobre um fenômeno a ser investigado. ${ }^{10}$

A metassíntese então se torna um conjunto de técnicas que tem a capacidade de ser indutiva e interpretativa, partindo dos casos específicos e chegando-se à síntese por meio da tradução recíproca. ${ }^{11}$

\section{Processo de busca e critérios de inclusão}

Foi realizada uma busca sistemática para identificar estudos de natureza qualitativa em bases de dados eletrônicas. Para selecionar as bases de dados que seriam utilizadas no estudo, foram levados em consideração os seguintes atributos: abrangência em termos de publicações, qualidade científica reconhecida no meio acadêmico e a característica das suas publicações consonantes ao tipo e objetivo da presente pesquisa. Desse modo, as bases utilizadas na pesquisa foram Scopus e PsycINFO (Figura 1).

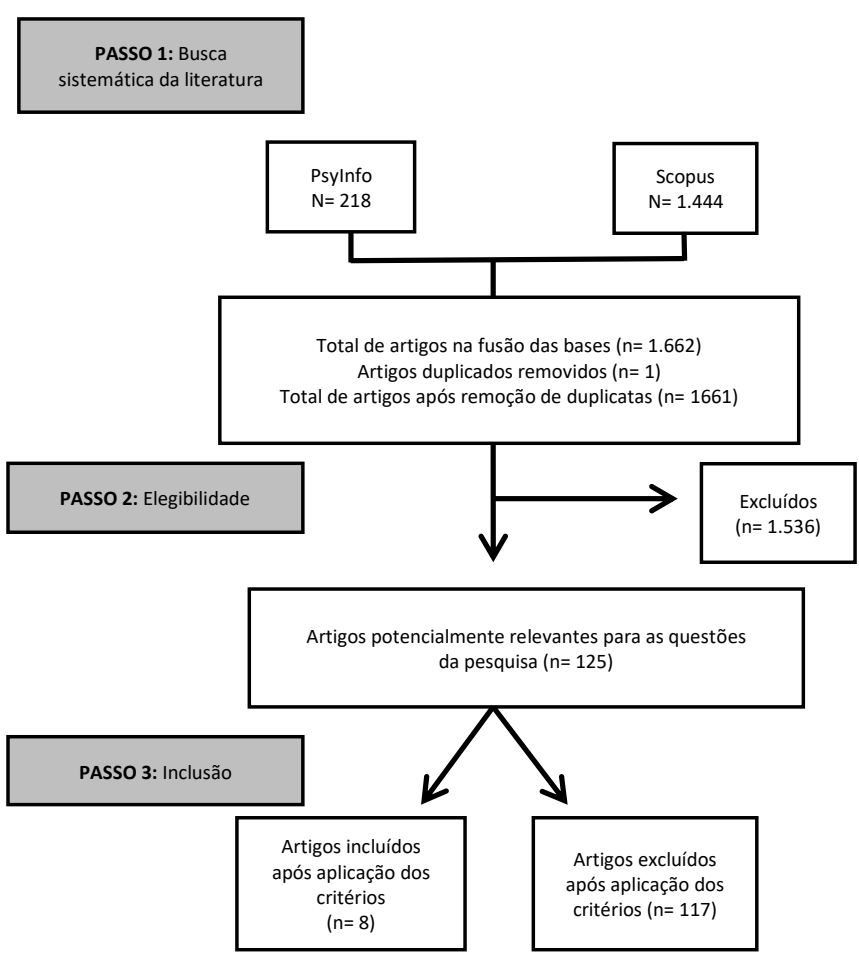

Figura 1. Processo de busca da literatura, elegibilidade e inclusão dos estudos

Foram selecionados artigos que continham os seguintes descritores ou seus correlatos: pesquisa qualitativa, crianças com deficiência, fisioterapia e pais, nos idiomas inglês e português (Quadro 1).

A seleção dos estudos para essa pesquisa envolveu os seguintes critérios de inclusão: a) estudos qualitativos; b) publicados em texto completo em revista/jornal revisado por pares entre os anos de 2013 a 2018; c) estudos que abordassem a percepção dos pais acerca do tratamento fisioterapêutico de crianças com DM. Todos os artigos 
foram lidos e selecionados por dois pesquisadores e em casos de dúvida sobre a inclusão de algum estudo um terceiro foi consultado para auxiliar nesse processo de seleção.

Os artigos potencialmente relevantes para atender a pergunta de pesquisa foram selecionados após a observação de etapas fundamentais, como: a) a realização de uma primeira leitura do título e resumo dos artigos que resultaram da busca; b) uma segunda leitura do texto completo daqueles artigos selecionados na etapa "a" aplicando os critérios de inclusão estabelecidos.

Quadro 1. Processo de pesquisa: descritores e seus correlatos

\begin{tabular}{|c|c|}
\hline $\begin{array}{l}\text { CRIANÇAS COM } \\
\text { DEFICIÊNCIA }\end{array}$ & $\begin{array}{l}\text { Português: Crianças portadoras de } \\
\text { deficiência OR Crianças com } \\
\text { deficiência } \\
\text { Inglês: Disabled children OR Children } \\
\text { with disability OR Disability, children } \\
\text { with }\end{array}$ \\
\hline $\begin{array}{l}\text { TRATAMENTO } \\
\text { FISIOTERAPÊUTICO }\end{array}$ & $\begin{array}{l}\text { Português: Fisioterapia OR } \\
\text { Reabilitação pediátrica OR } \\
\text { Reabilitação } \\
\text { Inglês: Physiotherapy OR Pediatric } \\
\text { rehabilitation OR Rehabilitation }\end{array}$ \\
\hline $\begin{array}{l}\text { METODOLOGIA } \\
\text { QUALITATIVA }\end{array}$ & $\begin{array}{l}\text { Português: Qualitativo OR } \\
\text { Abordagem narrativa OR Entrevista } \\
\text { com história de vida OR Entrevista } \\
\text { episódica OR Análise narrative OR } \\
\text { Estudo de caso OR Estudos de caso } \\
\text { OR Entrevistas semiestruturadas OR } \\
\text { Interpretativas OR Análise de discurso } \\
\text { OR Teoria Fundamentada OR Método } \\
\text { qualitativo OR Estudo qualitativo OR } \\
\text { Análise de conteúdo OR Percepção. } \\
\text { Inglês: Qualitative OR Narrative } \\
\text { approach OR Life-story interview OR } \\
\text { Episodic interview OR Narrative } \\
\text { analysis, OR Case studies OR Case } \\
\text { study OR Semi-structured interviews } \\
\text { OR Interpretative OR } \\
\text { Phenomenological analysis OR } \\
\text { Interviews OR Focus group OR } \\
\text { Discourse analysis OR Grounded } \\
\text { theory OR Qualitative method OR } \\
\text { Qualitative study OR Content analysis } \\
\text { OR Perception }\end{array}$ \\
\hline PAIS & $\begin{array}{l}\text { Português: Família OR Pais } \\
\text { Inglês: Family OR Parents }\end{array}$ \\
\hline
\end{tabular}

Avaliação da qualidade dos estudos

Após o processo de busca e elegibilidade, todos os estudos selecionados foram submetidos a uma avaliação crítica por meio do Consolidated Criteria for Reporting Qualitative Research(COREQ) que consiste em um relatório de identificação, desenvolvido para avaliar estudos qualitativos.

Ele é composto por uma série de itens que abrangem os desenhos dos estudos qualitativos, auxiliando os pesquisadores a relatar aspectos importantes encontrados relacionados à equipe de pesquisa, métodos de estudo, contexto do estudo, resultados, análise e interpretações. ${ }^{12}$ Ao todo, o instrumento possui 32 itens que são divididos em três domínios: 1) Equipe de pesquisa e reflexividade; 2 ) Desenho de estudo e 3) Análise dos achados. Com base nessa avaliação foram incluídos os artigos que alcançassem a maior pontuação nos itens compreendidos em cada domínio do COREQ. Assim, todos os oito estudos selecionados foram incluídos (Quadro 2).
Quadro 2. Avaliação do rigor metodológico segundo o Consolidated Criteria for Reporting Qualitative Research (COREQ) ${ }^{12}$

\begin{tabular}{|c|c|c|c|c|c|c|c|c|c|}
\hline & 1 & 2 & 3 & 4 & 5 & 6 & & & 8 \\
\hline \multicolumn{10}{|l|}{$\begin{array}{l}\text { Domínio 1: equipe de pesquisa e } \\
\text { reflexividade }\end{array}$} \\
\hline 1. Entrevistador / facilitador & $\mathrm{x}$ & $\mathrm{x}$ & $x$ & $x$ & & $x$ & & & $x$ \\
\hline 2. Credenciais & $x$ & & & $x$ & $x$ & & & & \\
\hline 3. Ocupação & $x$ & $\mathrm{x}$ & $x$ & $\mathrm{x}$ & & $x$ & & & $x$ \\
\hline 4. Gênero & & $\mathrm{x}$ & & & & & & & \\
\hline 5. Experiência / Treinamento & $x$ & & $x$ & & & & & & \\
\hline 6. Relacionamento com os participantes & & & $x$ & & & & & & \\
\hline $\begin{array}{l}\text { 7. Conhecimento dos participantes sobre a } \\
\text { intervenção }\end{array}$ & & $\mathrm{x}$ & $x$ & $x$ & $x$ & $x$ & & & $x$ \\
\hline 8. Características da intervenção & $x$ & $\mathrm{x}$ & $x$ & $x$ & & $x$ & & & $x$ \\
\hline \multicolumn{10}{|l|}{ Domínio 2: desenho de estudo } \\
\hline \multicolumn{10}{|l|}{ Quadro Teórico } \\
\hline 9. Metodologia de orientação & $x$ & $\mathrm{x}$ & $x$ & $x$ & $x$ & $x$ & & & $x$ \\
\hline 10. Amostragem & $x$ & $\mathrm{x}$ & $x$ & $x$ & $x$ & $x$ & & & $x$ \\
\hline 11. Método de abordagem & $\mathrm{x}$ & $x$ & $x$ & $x$ & $x$ & $\lambda$ & & & $x$ \\
\hline 12. Tamanho da amostra & $x$ & $\mathrm{x}$ & $x$ & $x$ & $x$ & $\lambda$ & & & $x$ \\
\hline 13. Não participação & & & $x$ & & & & & & $\mathrm{x}$ \\
\hline \multicolumn{10}{|l|}{ Configuração } \\
\hline 14. Configuração da coleta de dados & $x$ & $\mathrm{x}$ & $x$ & $x$ & $x$ & $\gamma$ & & & $x$ \\
\hline \multicolumn{10}{|l|}{ 15. Presença de não participantes } \\
\hline 16. Descrição da amostra & $x$ & & $x$ & $x$ & $x$ & $\gamma$ & & $x$ & $x$ \\
\hline \multicolumn{10}{|l|}{ A coleta de dados } \\
\hline 17. Guia de entrevista & $\mathbf{x}$ & $\mathrm{x}$ & $x$ & $x$ & $x$ & $x$ & & $\mathbf{x}$ & $x$ \\
\hline \multicolumn{10}{|l|}{ 18. Entrevistas repetidas } \\
\hline 19. Gravações áudio/visuais & $x$ & $\mathrm{x}$ & $x$ & $x$ & $x$ & $\gamma$ & & & $x$ \\
\hline 20. Notas de campo & $x$ & & & & & & & & \\
\hline 21. Duração & $x$ & $\mathrm{x}$ & $x$ & $x$ & & $x$ & & $x$ & \\
\hline 22. Saturação & & & $x$ & $x$ & $x$ & $x$ & & & \\
\hline \multicolumn{10}{|l|}{ 23. Transcrições refeitas } \\
\hline \multicolumn{10}{|l|}{ Domínio 3: análise dos achados } \\
\hline \multicolumn{10}{|l|}{ A análise de dados } \\
\hline 24. Número de dados coletados & $x$ & $\mathrm{x}$ & $x$ & $x$ & $x$ & $x$ & & & $x$ \\
\hline 25. Descrição dos dados coletados & $x$ & $x$ & $x$ & $x$ & $x$ & $x$ & & $x$ & $x$ \\
\hline 26. Derivação dos temas & $x$ & $\mathrm{x}$ & $x$ & $\mathrm{x}$ & $x$ & $\gamma$ & & $\mathbf{x}$ & $x$ \\
\hline 27. Software & $x$ & $\mathrm{x}$ & $x$ & & & & & & \\
\hline 28. Checagem dos participantes & $x$ & & & & & & & & \\
\hline \multicolumn{10}{|l|}{ Devolutiva } \\
\hline 29. Resultados apresentados & $x$ & $\mathrm{x}$ & $x$ & $\mathrm{x}$ & $x$ & $x$ & & $x$ & $x$ \\
\hline 30. Dados e resultados consistentes & $x$ & $\mathrm{x}$ & $x$ & $\mathrm{x}$ & $x$ & $\lambda$ & & $x$ & $x$ \\
\hline 31.Clareza dos temas maiores & $x$ & $\mathrm{x}$ & $x$ & $x$ & $x$ & $x$ & & $x$ & $x$ \\
\hline 32. Clareza dos temas menores & $x$ & $\mathrm{x}$ & $x$ & & & & & $\mathrm{x}$ & \\
\hline
\end{tabular}

Sequência dos artigos: 1. Evans-Rogers et al. ${ }^{13} ; 2$. Stefánsdóttir, Egilson ${ }^{14} ; 3$. Kruijsen-Terpstra et al. ${ }^{15} ; 4$. Jindal et al. ${ }^{16} ; 5$. Sukeri et al. ${ }^{17} ; 6$. Lillo-Navarro et al. ${ }^{18} ; 7$. Fitzgerald et al. ${ }^{19} ; 8$. Nakamanya et al. ${ }^{20}$

\section{Análise de dados}

De acordo com Noblit e Hare,$^{8}$ existem três estratégias que podem ser utilizadas para sintetizar estudos qualitativos: a Tradução Recíproca, onde os estudos são examinados em relação às 
semelhanças existentes entre eles; a Síntese de Refutação, utilizada quando o objetivo é identificar e analisar os desacordos existentes entre os estudos, e a Síntese de Linhas de Argumento, quando o interesse do estudo é o de desenvolver uma interpretação abrangente com base nas linhas de argumento elaborada nos sucessivos estudos.

Neste estudo foi utilizada a Tradução Recíproca como Síntese, tendo em vista que os estudos analisados possuíam diversas similaridades entre si, o que exige uma avaliação e comparação das ideias e conceitos presentes nos estudos originais.

0 processo de desenvolvimento de uma metassíntese envolve etapas de organização e análise de dados, os chamados conceitos de primeira, segunda e terceira ordem.

A primeira ordem envolveu a identificação dos conceitos relevantes provenientes dos principais achados dos artigos primários. $\mathrm{Na}$ interpretação de segunda ordem foi realizada a comparação entre os achados que surgiram em pelo menos dois estudos originais. A terceira ordem envolveu a reinterpretação dos conceitos de segunda ordem sintetizados em conceitos mais abrangentes, tendo como guia o objeto interesse da pesquisa. ${ }^{8}$

A metassíntese foi conduzida a partir das sete etapas fundamentais descritas por Noblit e Hare: ${ }^{8}$

(1) definição da área de interesse e a questão norteadora do caso estudado; (2) seleção dos estudos relevantes que farão parte da amostra; (3) releitura dos estudos e registros de informações, identificando as palavras-chaves, os temas e os conceitos centrais; (4) determinação da forma como os estudos estão relacionados; (5) comparação dos estudos entre si; (6) sintetização dos achados encontrados, privilegiando os achados mais recorrentes; (7) documentação e divulgação dos resultados da metassíntese.

\section{RESULTADOS}

O processo de busca e fusão dos resultados obtidos nas duas bases de dados resultou em 1.661 artigos. Realizando a leitura inicial de título e resumo, 125 artigos foram considerados potencialmente relevantes para o estudo, os demais foram excluídos, pois se tratavam de estudos quantitativos, a densa maioria, ou de revisão da literatura. Vale ressaltar que 117 artigos foram excluídos durante a leitura mais detalhada, visto que muitos deles abordaram apenas as deficiências intelectuais, não trataram do processo de reabilitação fisioterapêutica e/ou não relataram a percepção dos pais sobre os tratamentos realizados.

Os artigos incluídos eram de diversos países (Estados Unidos, Espanha, Malásia, Irlanda, Holanda, Sérvia, Uganda, Índia e Canadá), isso nos permitiu uma melhor observação das realidades socioculturais e familiares em diferentes lugares do mundo, o que enriquece os resultados encontrados.

No estudo em tela o fato de incluir realidades nacionais e culturais diferentes não resulta em dificuldade de análise, pois a metaetnografia na modalidade de tradução recíproca ${ }^{8}$ corresponde a uma interpretação da interpretação, tomando as semelhanças evidenciadas nos conceitos de primeira ordem, os quais são destacados em sua essência conforme o contexto sociocultural que os produziu e depois reunindo-os em uma síntese interpretativa que os representa, cujo objetivo é produzir aberturas para novas indagações e críticas. As principais características e conceitos de primeira ordem identificados nesses artigos estão representados no Quadro 3.

\section{Conceitos de segunda ordem}

Levando-se em consideração as questões de investigação, os principais pontos encontrados nos estudos resultaram nos cinco conceitos de segunda ordem descritos a seguir.

Relação de comunicação adequada e fornecimento de informações por parte dos profissionais
Um ponto importante descrito em três estudos foi o estabelecimento de um bom relacionamento e comunicação entre pais e profissionais. ${ }^{13-15}$ Os autores afirmam que a dedicação no cuidado, entusiasmo, respeito e a comunicação dos fisioterapeutas com os pais foram considerados como aspectos fundamentais. As qualidades pessoais dos terapeutas que incluem profissionalismo, serem atenciosos e dispostos a ouvir, buscar oferecer o melhor tratamento e encorajar os pais são importantes, pois refletem um bom relacionamento e contribuem para o sucesso do programa de tratamento.

A comunicação entre pais e terapeutas foi um tema abordado no estudo de Kruijsen-Terpstra al. ${ }^{15}$ apontando que os pais valorizaram os profissionais que tinham interesse em conversar sobre seus filhos e consideravam importante serem ouvidos e ter as suas opiniões levadas em conta no processo de reabilitação física. Assim, aponta-se como indispensável que os terapeutas interajam adequadamente com os pais para que uma relação de comunicação eficaz seja estabelecida. ${ }^{15}$

Por outro lado, alguns estudos identificaram que a falta de informações percebida pelos pais sobre o tratamento realizado e sobre a saúde em geral dos seus filhos era uma dificuldade existente no processo de reabilitação. ${ }^{15-17}$ Nestes trabalhos, os pais muitas vezes expressaram a necessidade de informações, especialmente no que diz respeito a três aspectos: conhecimento sobre o que é a doença que seus filhos têm, o tratamento realizado com eles e quais são as perspectivas que podem esperar para o futuro. ${ }^{15-16}$

Um estudo verificou que os pais achavam difícil pedir informações específicas no momento em que ainda não estavam familiarizados com o diagnóstico dos seus filhos e também com o ambiente de reabilitação. Então, valoriza-se quando os profissionais tomam a iniciativa de fornecer esse tipo de informação. ${ }^{15}$

Além disso, dois autores destacaram o fato de que muitos pais relataram não estarem cientes do que vinha sendo realizado com seus filhos durante os atendimentos fisioterapêuticos. Para os pais essa informação era relevante para que se tornassem capazes de escolher juntamente com os fisioterapeutas quais seriam as melhores intervenções para seus filhos.

Além disso, vislumbravam a possibilidade de que essa construção compartilhada do processo fisioterapêutico os instrumentalizasse de alguma forma, para realizar exercícios em casa que contribuíssem no processo de reabilitação e favorecessem uma maior funcionalidade e independência. ${ }^{15,16}$

Outro aspecto citado na literatura utilizada nesta metassintese diz respeito ao desejo que a maioria dos pais possuía de saber o que poderiam de fato esperar para o futuro dos seus filhos, pois muitos acabavam se decepcionando por não ver evolução no quadro e outros não tinham mais expectativas de melhoras. ${ }^{15,16}$

Sukeri et al. ${ }^{17}$ afirmam que os pais se sentiam privados de informações cruciais para melhorar a qualidade de vida de seus filhos, resultando em necessidades que não eram adequadamente atendidas e retardo na busca de tratamento necessário. Também destacam que o fornecimento de informações claras pode capacitar os pais de crianças com deficiência, dando-Ihes o conhecimento e a habilidade necessários para tomar decisões apropriadas, aumentar o sentimento de autonomia e diminuir os sentimentos de impotência, medo, ansiedade, incerteza e estresse.

Falta de incentivo por parte dos terapeutas para que os pais participem da terapia

Os estudos apontaram outro déficit no processo de reabilitação: a falta de incentivo para que os pais também participem da terapia juntamente com seus filhos e os terapeutas que os atendem. ${ }^{14,15}$

No estudo de Stefánsdóttir e Egilson, ${ }^{14}$ três tipos de pais foram percebidos na análise das entrevistas: 1 . Pais que se consideravam leigos; 2 . Pais que se consideravam especialistas com relação aos seus filhos; 3 . Pais que estavam em algum lugar entre os dois anteriores. 
Quadro 3. Artigos selecionados e descrição detalhada do conceitos de primeira ordem

\begin{tabular}{|c|c|c|c|c|c|}
\hline $\begin{array}{l}\text { Autor } \\
\text { (País) }\end{array}$ & Objetivos & Participantes & $\begin{array}{c}\text { Coleta } \\
\text { de dados }\end{array}$ & $\begin{array}{c}\text { Método } \\
\text { de análise }\end{array}$ & $\begin{array}{c}\text { Conceitos de } \\
\text { primeira ordem }\end{array}$ \\
\hline $\begin{array}{l}\text { Evans-Rogers et al. }{ }^{13} \\
\text { EUA }\end{array}$ & $\begin{array}{l}\text { Investigar as percepções } \\
\text { parentais sobre a } \\
\text { participação de seus } \\
\text { filhos em um programa } \\
\text { de intervenção NDT e } \\
\text { examinar } \\
\text { quantitativamente as } \\
\text { diferenças nas } \\
\text { habilidades funcionais de } \\
\text { crianças com PC e outras } \\
\text { disfunções neuromotoras }\end{array}$ & $\begin{array}{l}12 \text { mães e } 1 \text { avó } \\
\text { de crianças com } \\
\text { diagnóstico de } \\
\text { paralisia cerebral } \\
\text { ou outra } \\
\text { condição } \\
\text { neuromotora que } \\
\text { afeta a } \\
\text { participação e } \\
\text { habilidades } \\
\text { funcionais }\end{array}$ & $\begin{array}{l}\text { Entrevista } \\
\text { semiestruturada }\end{array}$ & $\begin{array}{l}\text { Abordagem } \\
\text { fenomenológica e } \\
\text { método quase- } \\
\text { experimental }\end{array}$ & $\begin{array}{l}\text { - Atendeu as necessidades das } \\
\text { crianças; } \\
\text { - Sentimentos de esperança e } \\
\text { motivação ao ver melhorias funcionais; } \\
\text { - Terapeutas solícitos e disponíveis } \\
\text { para escutar os pais e as crianças; } \\
\text { - Explicações sobre manuseios } \\
\text { oferecidas pelo terapeuta; } \\
\text { - Trabalho em equipe e envolvendo } \\
\text { pais e crianças; } \\
\text { - Exercícios terapêuticos domiciliares } \\
\text { foram fundamentais; } \\
\text {-Importância de cum roteiro de } \\
\text { exercícios para guiar os pais; } \\
\text { - Dificuldade para pagar terapias de } \\
\text { reabilitação }\end{array}$ \\
\hline $\begin{array}{l}\text { Stefánsdóttir, Egilson }{ }^{14} \\
\text { Sérvia }\end{array}$ & $\begin{array}{l}\text { Examinar em que medida } \\
\text { os pais e terapeutas } \\
\text { percebem o serviço como } \\
\text { centrado na família; } \\
\text { explorar os fatores que } \\
\text { afetam a percepção dos } \\
\text { pais sobre o serviço; } \\
\text { explorar como os pais } \\
\text { entendiam os aspectos } \\
\text { do serviço que o } \\
\text { caracterizava como } \\
\text { centrado na família }\end{array}$ & $\begin{array}{l}\text { Pais de } 236 \\
\text { crianças que } \\
\text { receberam } \\
\text { atendimento no } \\
\text { centro de } \\
\text { reabilitação } \\
\text { durante um } \\
\text { período de seis } \\
\text { meses e } 25 \\
\text { fisioterapeutas e } \\
\text { terapeutas } \\
\text { ocupacionais }\end{array}$ & $\begin{array}{l}\text { Entrevista } \\
\text { individual }\end{array}$ & $\begin{array}{l}\text { Análise } \\
\text { quantitativa e } \\
\text { qualitativa e } \\
\text { análise descritiva } \\
\text { de conteúdo }\end{array}$ & $\begin{array}{l}\text { - Importância do respeito por parte dos } \\
\text { profissionais e a confiança mútua pais- } \\
\text { terapeutas; } \\
\text { - Falta de incentivo em participar do } \\
\text { atendimento juntamente com seus } \\
\text { filhos; } \\
\text { - Definição compartilhada de metas de } \\
\text { tratamento melhorou os resultados da } \\
\text { terapia; } \\
\text { - Pais valorizaram os benefícios da } \\
\text { terapia }\end{array}$ \\
\hline $\begin{array}{l}\text { Kruijsen-Terpstra et al. }{ }^{15} \\
\text { Holanda }\end{array}$ & $\begin{array}{l}\text { Explorar as experiências e } \\
\text { necessidades de pais de } \\
\text { crianças pequenas (entre } \\
2 \text { e } 4 \text { anos de idade) com } \\
\text { paralisia cerebral sobre o } \\
\text { serviço de fisioterapia e } \\
\text { terapia ocupacional } \\
\text { ofertados a seus filhos } \\
\text { em um ambiente de } \\
\text { reabilitação }\end{array}$ & $\begin{array}{l}21 \text { famílias de } \\
\text { crianças com } \\
\text { paralisia cerebral } \\
\text { (em duas famílias } \\
\text { pais e mães } \\
\text { estavam } \\
\text { presentes e nas } \\
\text { demais apenas a } \\
\text { mãe foi } \\
\text { entrevistada) }\end{array}$ & $\begin{array}{l}\text { Entrevista } \\
\text { semiestruturada }\end{array}$ & Análise temática & $\begin{array}{l}\text { - Falta de informações sobre a saúde } \\
\text { dos filhos; } \\
\text { - Importância do fornecimento de } \\
\text { informações por parte dos } \\
\text { profissionais; } \\
\text { - Importância da relação de } \\
\text { comunicação com os terapeutas; } \\
\text { - Alguns pais sentiram falta de serem } \\
\text { escutados e encorajados; } \\
\text { - Construção de parceria entre } \\
\text { terapeutas e pais na definição das } \\
\text { metas de tratamento de seus filhos; } \\
\text { - Terapeutas não estimulavam o } \\
\text { envolvimento dos pais na terapia }\end{array}$ \\
\hline $\begin{array}{l}\text { Jindal et al. }{ }^{16} \\
\text { Índia e Canadá }\end{array}$ & $\begin{array}{l}\text { Explorar as perspectivas } \\
\text { de pais com diferentes } \\
\text { condições } \\
\text { socioeconômicas e } \\
\text { culturais sobre a } \\
\text { reabilitação de seus filhos } \\
\text { com paralisia cerebral } \\
\text { (PC) e as necessidades de } \\
\text { informação dos pais em } \\
\text { relação à reabilitação de } \\
\text { seus filhos com PC }\end{array}$ & $\begin{array}{l}18 \text { pais de } \\
\text { crianças com } \\
\text { Paralisia Cerebral } \\
\text { (11 do Canadá e } \\
7 \text { da Índia) }\end{array}$ & $\begin{array}{l}\text { Entrevista } \\
\text { semiestruturada }\end{array}$ & Análise temática & $\begin{array}{l}\text { - Pais acreditavam que a fisioterapia } \\
\text { seria capaz de melhorar a } \\
\text { funcionalidade da criança; } \\
\text { - Falta de informações por parte dos } \\
\text { profissionais sobre o processo de } \\
\text { reabilitação dos filhos }\end{array}$ \\
\hline $\begin{array}{l}\text { Sukeri et al. }{ }^{17} \\
\text { Malásia }\end{array}$ & $\begin{array}{l}\text { Explorar as barreiras } \\
\text { existentes para a } \\
\text { realização de } \\
\text { necessidades não } \\
\text { satisfeitas entre mães de } \\
\text { crianças com deficiência }\end{array}$ & $\begin{array}{l}13 \text { mães de } \\
\text { crianças que } \\
\text { apresentam } \\
\text { deficiência física }\end{array}$ & $\begin{array}{l}\text { Entrevista } \\
\text { individual }\end{array}$ & Análise temática & $\begin{array}{l}\text { - Falta de comunicação entre pais e } \\
\text { profissionais de saúde; } \\
\text { - Pouca informação sobre o tratamento } \\
\text { realizado; } \\
\text { - Falta de ajuda financeira e para o } \\
\text { transporte; } \\
\text { - Escassez de apoio social }\end{array}$ \\
\hline $\begin{array}{l}\text { Lillo-Navarro et al. }{ }^{18} \\
\text { Espanha }\end{array}$ & $\begin{array}{l}\text { Investigar as percepções } \\
\text { dos pais sobre os } \\
\text { exercícios domiciliares } \\
\text { prescritos pelos } \\
\text { fisioterapeutas para } \\
\text { crianças com deficiência }\end{array}$ & $\begin{array}{l}28 \text { Pais de } \\
\text { crianças com } \\
\text { deficiência que } \\
\text { tinham recebido } \\
\text { um programa de } \\
\text { exercícios } \\
\text { domiciliares } \\
\text { prescritos por } \\
\text { fisioterapeutas }\end{array}$ & Grupos focais & $\begin{array}{l}\text { Teoria } \\
\text { fundamentada } \\
\text { nos dados }\end{array}$ & $\begin{array}{l}\text { - Motivação para realizar exercícios } \\
\text { quando percebem que eles funcionam } \\
\text { e são eficazes; } \\
\text { - Fisioterapeutas ajudaram a construir } \\
\text { a confiança dos pais; } \\
\text {-Utilidade de cartilhas de exercícios } \\
\text { para guia-los; } \\
\text { - Interação entre pais e fisioterapeutas } \\
\text { na adesão aos exercícios }\end{array}$ \\
\hline
\end{tabular}




\begin{tabular}{|c|c|c|c|c|c|}
\hline $\begin{array}{l}\text { Fitzgerald et al. }{ }^{19} \\
\text { Irlanda }\end{array}$ & $\begin{array}{l}\text { Explorar as experiências } \\
\text { dos pais e identificar } \\
\text { quaisquer problemas } \\
\text { com os serviços } \\
\text { existentes, que podem } \\
\text { apoiar o } \\
\text { desenvolvimento de } \\
\text { estratégias eficientes e } \\
\text { eficazes para ajudar as } \\
\text { crianças com deficiência } \\
\text { no futuro }\end{array}$ & $\begin{array}{l}14 \text { mães e três } \\
\text { pais de famílias } \\
\text { diferentes, assim } \\
\text { como uma mãe e } \\
\text { um pai da mesma } \\
\text { família }\end{array}$ & $\begin{array}{l}\text { Entrevista } \\
\text { semiestruturada } \\
\text { e narrativa livre }\end{array}$ & $\begin{array}{l}\text { Teoria } \\
\text { fundamentada } \\
\text { nos dados }\end{array}$ & $\begin{array}{l}\text { - Abordagem centrada na criança } \\
\text { melhorou o desenvolvimento; } \\
\text { - Trabalho em equipe foi positivo; } \\
\text { Serviços prestados inadequados; } \\
\text { - Boa relação entre pais e a equipe } \\
\text { multidisciplinar; } \\
\text { - Trabalho em conjunto da equipe com } \\
\text { os pais }\end{array}$ \\
\hline $\begin{array}{l}\text { Nakamanya et al. }{ }^{20} \\
\text { Uganda }\end{array}$ & $\begin{array}{l}\text { Explorar experiências } \\
\text { maternas de cuidar de } \\
\text { uma criança afetada por } \\
\text { atraso no } \\
\text { neurodesenvolvimento } \\
\text { após encefalopatia } \\
\text { neonatal }\end{array}$ & $\begin{array}{l}15 \text { mães e um } \\
\text { cuidador de } \\
\text { crianças com } \\
\text { encefalopatia }\end{array}$ & $\begin{array}{l}\text { Entrevista em } \\
\text { grupo e } \\
\text { individual }\end{array}$ & $\begin{array}{l}\text { Análise de } \\
\text { conteúdo }\end{array}$ & $\begin{array}{l}\text { - Mães reagiram positivamente à } \\
\text { fisioterapia; } \\
\text { - Falta de dinheiro para o tratamento e } \\
\text { transporte; } \\
\text { - Escassez de apoio social }\end{array}$ \\
\hline
\end{tabular}

Os pais que se percebiam como leigos não se sentiam chamados a se envolver na terapia e preferiam deixar o trabalho exclusivamente para os terapeutas.

Conforme descrito pelos autores, os pais que se consideravam "especialistas", queriam estar altamente envolvidos nas intervenções com seus filhos, porém relataram que os terapeutas às vezes os fizeram sentir que não seria uma boa ideia essa participação.

Outros afirmam que estariam mais satisfeitos se os profissionais os tivessem envolvido no tratamento. ${ }^{18}$ Por fim, são citados os pais que se consideraram em algum lugar entre leigos e especialistas e os autores mostram que esses pais gostariam de estar mais envolvidos, mas muitas vezes não se sentem capazes e incentivados. ${ }^{14}$

No estudo de Kruijsen-Terpstra et al. ${ }^{15}$ ficou claro que em várias ocasiões o papel dos pais no processo de reabilitação não foi bem definido e muitos deles costumavam apenas sentar na sala de espera e ler revistas enquanto seus filhos eram atendidos. Porém, isso não se devia ao fato de eles não quererem se envolver e sim porque não eram estimulados para isso e não recebiam informações adequadas por parte dos terapeutas sobre o tratamento realizado, com isso não conheciam qual era o seu papel nesse cenário.

A ausência dos pais durante o atendimento representa uma dificuldade para que os terapeutas ajustem o tratamento dos pacientes às suas necessidades, dificulta o seu processo de capacitação para lidar com a doença dos filhos e limita o estabelecimento de uma parceria, que é imprescindível nesse processo. $^{14}$

O trabalho de parceria entre os pais, o fisioterapeuta e demais membros da equipe de reabilitação na definição das metas de tratamento

Os estudos mostram que os pais veem como aspecto positivo a interação e o trabalho em equipe desenvolvido pelos terapeutas. Os autores relatam que essa cooperação e trabalho conjunto entre os membros da equipe foi algo muito valorizado, especialmente quando os pais também estavam envolvidos nesse processo, pois eles sentiam que desse modo existia uma relação de trabalho em conjunto, onde todos contribuíam para maior eficácia do tratamento. ${ }^{13,19}$

Desenvolver uma relação de trabalho conjunto com a equipe facilita o processo de inclusão dos pais na terapia e torna a convivência entre eles mais agradável. ${ }^{19}$ Nos estudos de Evans Rogers et al. ${ }^{13} \mathrm{e}$ Fitzgerald et al. ${ }^{19}$ fica claro que os pais perceberam quando esse trabalho em equipe de fato acontecia e o quanto eles consideravam valioso o diálogo e a cooperação entre os profissionais na busca de decidir os melhores caminhos a serem seguidos no tratamento dos seus filhos. Além disso, os três autores que abordaram essa temática mostraram que os pais consideram de extrema importância a construção de parceria e de comunicação entre eles e os terapeutas para definir os objetivos e metas de tratamento dos seus filhos. ${ }^{13,15,19}$
A definição compartilhada de metas foi capaz de promover a colaboração e a coordenação entre as famílias e o centro de reabilitação e levou a melhores resultados de intervenção. ${ }^{19}$ Também foi demonstrado como aspecto positivo o fato de que os objetivos e metas, quando definidos em conjunto, refletiam o que os pais tinham percebido como importante e o que os terapeutas também achavam que seria importante trabalhar. ${ }^{15}$

No estudo de Fitzgerald et al. ${ }^{19}$ fica claro que a abordagem transdisciplinar foi capaz de incluir e empoderar a família das crianças e isso significava que a equipe e os pais trabalharam em conjunto para atender as necessidades das crianças através da criação de objetivos e metas de tratamento.

\section{Exercícios terapêuticos domiciliares e auxílio dos terapeutas aos pais para favorecer a realização}

Dois autores trouxeram em seus estudos outro aspecto relevante: a percepção dos pais quanto à realização de exercícios em casa com seus filhos sob a orientação dos terapeutas. ${ }^{13,18}$ Essa questão foi considerada fundamental na obtenção do sucesso em um programa de intervenção com o tratamento neuroevolutivo, pois tornaria possivel aos pais ocupar uma parte do tempo em casa realizando os exercícios com seus filhos, sendo orientados por uma cartilha preparada pelos profissionais, dando continuidade ao tratamento iniciado no centro de reabilitação e assim favorecer uma evolução mais significativa dessas crianças. ${ }^{13}$

Lillo Navarroet al. ${ }^{18}$ ao investigar as percepções dos pais sobre os exercícios domiciliares prescritos pelos fisioterapeutas para seus filhos, identificaram que os pais se sentiam confiantes para realizar os exercícios domiciliares quando os fisioterapeutas os auxiliavam e forneciam instruções sobre o que deveria ser realizado. Além disso, os autores mostram que alguns pais realmente percebiam a importância dos exercícios domiciliares, conseguiam observar melhorias funcionais dos seus filhos e acreditavam que esses exercícios ajudaram nessas conquistas.

\section{Observação de melhorias físicas nas crianças}

Quatro estudos analisados abordaram a percepção dos pais sobre as melhorias físicas que seus filhos adquiriram desde quando iniciaram o tratamento fisioterapêutico. ${ }^{13,14,16,20}$ Diversas melhorias foram relatadas pelos pais, como o aumento da flexibilidade, da força muscular, melhora do tônus e do equilíbrio, progresso na mobilidade (atividades de rolar, sentar, engatinhar e ficar em pé) e independência durante atividades diárias. ${ }^{20,21}$

No estudo de Stefánsdóttir e Egilson ${ }^{14}$ o tratamento fisioterapêutico foi percebido como benéfico e por isso os pais desejavam que esse tipo de serviço também fosse incluído e integrado no ambiente cotidiano das crianças buscando favorecer um melhor 
desenvolvimento delas em outros espaços (especialmente nas escolas).

Evans Rogers et al. ${ }^{13}$ relataram que os pais eram tomados por sentimentos de esperança e motivação ao perceberem as melhoras que os seus filhos tinham conquistado.

\section{DISCUSSÃO}

Levando-se em consideração os conceitos de segunda ordem anteriormente descritos, foi possível elaborar duas sínteses. A primeira diz respeito à participação dos pais no processo de reabilitação e a segunda, como os pais percebem as melhorias físicas dos seus filhos em decorrência do tratamento e a sua comunicação com fisioterapeutas.

\section{Participação dos pais no processo de reabilitação}

Os conceitos de segunda ordem que deram origem a essa síntese foram: 1) Falta de incentivo por parte dos profissionais para que os pais participem da terapia; 2) O trabalho em equipe envolvendo os pais e a parceria na definição das metas de tratamento; e 3) Exercícios terapêuticos domiciliares e auxílio dos profissionais aos pais para favorecer a realização.

Analisar como se dá a participação dos pais no tratamento de reabilitação quanto a melhora dos seus filhos é algo relevante e ainda pouco explorado, mesmo a despeita de evidências de que os resultados do tratamento parecem melhores quando os pais participam do processo de reabilitação. ${ }^{22-24} A$ atuação conjunta entre a família e os profissionais resulta em benefícios essenciais para o desenvolvimento da criança em tratamento, no que diz respeito, entre outros, ao seu crescimento e desenvolvimento integral. ${ }^{21}$

Rinaldi, ${ }^{25}$ afirma que o modelo de intervenção funcional centrado na família a prevê como parceira da equipe de reabilitação e, como tal, ela deve ter participação ativa nas tomadas de decisões. Portanto, esse modelo tem uma abordagem que vai além da capacidade individual da criança, amplia a intervenção para que esta possa também englobar o ambiente e os objetivos da criança e da sua família, assim como suas habilidades físicas.

Uchôa et al. ${ }^{26}$ cita pontos positivos resultantes do trabalho em equipe, como por exemplo, "a possibilidade de formar uma rede de relações entre as pessoas, rompendo a visão dominante e individualista das especialidades, permitindo manter uma união e troca de ideias, saberes, vontade e trabalho voltados às práticas inovadoras."

Outro ponto destacado foi a importância da parceria desenvolvida entre pais e fisioterapeutas para a definição dos objetivos e metas de tratamento dos seus filhos. De acordo com Rotta ${ }^{27}$ e Izumi ${ }^{28}$ a programação terapêutica deve ser elaborada de acordo com as necessidades da criança, em conjunto com seus pais, pois a intervenção tem sido mais benéfica para as crianças quando os pais participam do tratamento juntamente com o terapeuta.

Nesse processo de inclusão dos pais, é importante que os fisioterapeutas sejam sensíveisao envolvimento da família e ao conhecimento das suas necessidades relacionadas com o sucesso do tratamento realizado. Os pais são importantes no processo de avaliar, educar e reabilitar as crianças com deficiência ${ }^{29}$ e é possível observar que oprocesso de articulação entre pais e terapeutas na definição dos objetivos e metas de tratamento proporciona uma maior integração entre a criança, sua rede familiar e o profissional responsável pelo seu cuidado e isso facilita a escolha de metas que reflitam a real necessidade da criança e também aquilo que os pais percebem que é importante trabalhar, com apoio do terapeuta, durante a reabilitação.

Por outro lado, nos estudos analisados foi possível perceber que existe uma falta de incentivo por parte dos terapeutas para que os pais também participem do processo de reabilitação. ${ }^{14,15} \mathrm{~A}$ pouca participação no tratamento é uma das dificuldades mais encontradas na prática de reabilitação. Nesse sentido, torna-se necessária uma atuação mais efetiva por parte dos profissionais através do esclarecimento dos objetivos do tratamento fisioterapêutico, o maior incentivo para a participação na escolha dos métodos de tratamento e uma melhor conscientização dos pais sobre por que de fato se torna importante a sua participação nesse processo. ${ }^{21}$

A partir dessas atitudes se tornará possível construir um ambiente mais favorável à inserção dos pais no tratamento e assim poderão ser alcançados resultados mais satisfatórios com a reabilitação.

Além desse aspecto, um estudo também evidenciou que alguns pais não se sentiam chamados a participar do tratamento e preferiam deixar o trabalho exclusivamente para os terapeutas. Isso demonstra que outros fatores podem influenciar a participação da família da criança no processo de reabilitação.

Um deles diz respeito à falta de conhecimento dos pais sobre a patologia e o tratamento fisioterapêutico, o que os impede de assumir um papel mais ativo na reabilitação. ${ }^{30}$ Outro fator é a falta de tempo, uma vez que por serem as mães as mais envolvidas no cuidado, muitas são sobrecarregadas com os trabalhos domésticos e ainda por terem que trabalhar fora de casa e isso impossibilita um maior compromisso e dedicação delas com a reabilitação dos filhos.

Por fim, um outro obstáculo à participação é a perda de expectativas de melhora da criança, pois à medida que as crianças crescem e os familiares, especialmente as mães, percebem que a recuperação é muito lenta e a evolução do quadro não é a esperada, a participação no tratamento se torna infrequente e esta responsabilidade passa a ser creditada apenas à instituição. ${ }^{31}$

Diversos estudos mostram que a prática de exercícios domiciliares é fundamental para se alcançar uma maior evolução da criança e otimizar o seu desenvolvimento neuropsicomotor. ${ }^{21,32} \mathrm{~A}$ importância de manter os pais presentes no tratamento se dá pela necessidade de levar para o cotidiano da criança as habilidades construídas durante as sessões de fisioterapia. ${ }^{31}$

Os exercícios domiciliares foram considerados fundamentais para obtenção de melhores resultados com o tratamento. Concordando com esses achados, Lima, ${ }^{31}$ Barbosa et al. ${ }^{32}$ e Lewandovski e Carrilho ${ }^{33}$ mostraram que grande parte dos pais afirmou que a continuidade do tratamento no domicílio ajuda na recuperação, algumas mães conseguiam perceber claramente a necessidade de dar continuidade aos exercícios em casa e outras reconheceram que pelo fato das crianças passarem a maior parte do tempo em casa, assimé necessário que sejam bem assistidas para conseguir êxito no tratamento como um todo.

Sari e Marcon ${ }^{21}$ afirmam que a família deve ser estimulada e incentivada para a realização dos exercícios domiciliares, pois o ambiente mais apropriado, o carinho e a atenção da família são capazes de reforçar o vínculo com a criança, o que então favorece seu maior desenvolvimento.

Além disso, a realização de exercícios domiciliares permite que a criança receba uma quantidade maior de estímulos, fundamentais para a evolução do tratamento. A família necessita do apoio profissional, de orientação e também de ter suas dúvidas e necessidades levadas em consideração, esse aspecto fica bem evidente quando nos estudos avaliados nessa pesquisa os pais afirmam que se sentiam confiantes para realizar os exercícios quando os terapeutas os auxiliavam e forneciam instruções sobre o que deveria ser feito com seus filhos. ${ }^{24}$

\section{Informação e comunicação entre pais e fisioterapeutas na percepção das melhoras físicas obtidas com o tratamento}

Essa síntese foi elaborada a partir de dois conceitos de segunda ordem: 1) Relação de comunicação adequada e fornecimento de informações por parte dos profissionais e 2) Observação de melhorias físicas nas crianças.

A atuação fisioterapêutica na pediatria é imprescindível para favorecer à criança a obtenção de habilidades motoras e sua melhor interação com o ambiente, além de prevenir deformidades e 
contraturas que podem prejudicar sua qualidade de vida. ${ }^{6}$ Dentre os principais objetivos de tratamento estão a estimulação do aprendizado e do controle motor e o aprimoramentoda funcionalidade na criança.

Nos artigos investigados, os pais classificaram a reabilitação como benéfica, sendo relatadas melhoras significativas no quadro clínico das crianças. Diversos estudos que analisaram a concepção dos pais sobre a fisioterapia no tratamento de crianças com deficiência, evidenciaram que, na visão da grande maioria dos pais, a fisioterapia é importante no processo evolutivo de seus filhos, sendo uma ajuda que possibilita uma maior autonomia, além de favorecer um maior desenvolvimento motor e inclusão dessas crianças na sociedade. ${ }^{21,25,31,33,34}$

Izumi $^{28}$ ao entrevistar pais de crianças com deficiência neuromotora também encontrou resultados positivos quanto ao tratamento fisioterapêutico pois os pais tinham confiança no tratamento proposto e as mães viam a fisioterapia como um tratamento fundamental para que seus filhos obtivessem aquisições motoras, o que favoreceria uma maior qualidade de vida e independência funcional.

Pequenos ganhos de desenvolvimento da criança com DM são capazes de renovar as esperanças maternas. ${ }^{31} \mathrm{O}$ sentimento de alegria é vivenciado de forma intensa pelas mães ao perceberem que seus filhos conseguem comer sozinhos, sentam-se sozinhos ou mesmo são capazes de pegar um brinquedo com as próprias mãos. ${ }^{31}$ Por isso, Rinaldi ${ }^{25}$ alerta que o fisioterapeuta deve acolher as famílias, ouvi-las, prestar esclarecimentos e informações.

Nos estudos avaliados nessa metassíntese, foram mencionadas pelos pais diversas evoluções físicas, promovendo uma maior independência e qualidade de vida para essas crianças e suas famílias e estes resultados são ainda mais significativos quando a intervenção é iniciada precocemente, pois conforme frisado por Souza e Pires ${ }^{35}$ os benefícios da intervenção precoce nas crianças com deficiências e nas suas famílias vão além dos ganhos neuromotores porque a intervenção precoce possibilita que a criança consiga um maior desenvolvimento da sua condição e pode favorecer maior estabilidade e segurança para as crianças e suas famílias.

Nesse processo de reabilitação, fornecer orientações e informações para a família das crianças com deficiência é fundamental e, nesse cenário, o profissional de saúde desempenha o papel de auxiliar a família e favorecer o estabelecimento de uma comunicação adequada. Como um profissional intensamente envolvido no tratamento de crianças com deficiência, o fisioterapeuta deve ser capaz de conhecer quais são as prioridades e preferências da família para que assim possam oferecer instruções, orientações e recomendações que sejam úteis para auxiliar no cuidado. ${ }^{29}$

Alguns estudos analisados nessa metassíntese mostram que os pais valorizam os profissionais que se comunicam adequadamente com eles a respeito da saúde de seus filhos e do tratamento realizado. ${ }^{13-15}$ No entanto, outros trazem à tona o problema de falta de informações percebida pelos pais por parte dos profissionais. ${ }^{16,17} \mathrm{~A}$ literatura que aborda essa última temática evidencia que essa escassez de informações de fato está instalada e isso interfere negativamente no processo de reabilitação. ${ }^{29,31,33,36,37}$

Alguns pontos acerca dessa temática foram abordados nos estudos e um deles consistiu no pouco conhecimento dos pais sobre o diagnóstico e a doença dos filhos.

Sobre esse assunto, Araújo ${ }^{38}$ afirma que ainda existe uma falha no conhecimento das mães sobre a doença que seus filhos possuem, o que deixa claro a necessidade de melhorar a comunicação entre a equipe e a família, visto que alguns profissionais explicam de maneira superficial os pontos relacionados à doença e ao tratamento das crianças.

Gração e Santos ${ }^{39}$ afirmaram que um dos fatores necessários para a realização de um programa de tratamento é o esclarecimento das mães no que diz respeito à doença dos seus filhos e também sobre o tratamento proposto, para que elas possam desenvolver um papel importante na prestação de cuidados, tendo em vista que se tornam peças fundamentais durante o processo de reabilitação.

Contrastando com essa afirmação, Lewandosvski e Carrilho, ${ }^{33}$ alegam que as informações fornecidas pelos profissionais não estão sendo suficientes para que a família possa suprir as demandas de cuidados das crianças no ambiente domiciliar.

Em geral, as famílias se sentem pouco conhecedoras do estado de saúde da criança e, portanto, inseguras ao prestarem cuidados. Essa constatação harmoniza com os resultados obtidos nessa pesquisa onde os autores dos estudos mostram que os pais não estavam cientes do que vinha sendo realizado com seus filhos na terapia e muitas vezes desejavam esse tipo de informação para ter a capacidade de auxiliar no processo de reabilitação e favorecer uma maior funcionalidade e independência às crianças. A falta de orientações concretas pode levar a uma insegurança no cuidado com a criança e a família passa então a questionar a eficácia do tratamento. ${ }^{37}$

Por fim, outro ponto citado nos artigos foi o que diz respeito ao desejo que a maioria dos pais possuía de saber o que poderiam de fato esperar para o futuro dos seus filhos. Bernardo ${ }^{29}$ mostra que uma das necessidades mais descritas pelos pais é a de perceber a normalidade e também de obter alguma certeza sobre o futuro delas.

Desse modo, percebe-se que é fundamental o diálogo entre os fisioterapeutas e os pais sobre a condição de saúde dos seus filhos e o que pode ser alcançado com o tratamento, para que eles possam de fato compreender até onde a fisioterapia pode ir para proporcionar uma melhora da condição geral de saúde e assim sintam mais segurança quanto ao desenvolvimento e ao futuro das suas crianças.

\section{CONCLUSÃO}

Na percepção dos pais a fisioterapia assume um papel importante na melhora da condição clínica das crianças e é capaz de trazer diversos benefícios em relação ao desenvolvimento motor, além de proporcionar uma melhor qualidade de vida para a família em geral, conforme observado nos estudos analisados. No entanto, ainda existem pontos que influenciam negativamente na realização de um programa de reabilitação e que precisam ser levados em consideração, os quais foram descritos ao longo dessa pesquisa.

A falha na comunicação e fornecimento de informações aos pais sobre a saúde e o processo de reabilitação das crianças é um problema bastante comum e que acaba dificultando o estabelecimento de um bom relacionamento entre pais e terapeutas, assim como priva a família de receber orientações e instruções que podem ser de grande valia para obtenção de maior sucesso no tratamento.

Foi observado ainda que há muito o que ser melhorado no que se refere à participação efetiva dos pais no tratamento dos seus filhos. Nos estudos analisados apenas a falta de incentivo dos profissionais foi citada como ponto que mais interferiu na participação desses pais, porém consideramos que muitos outros elementos também podem estar envolvidos nesse cenário e merecem ser analisados com maior profundidade em estudos futuros.

Como limitação apontamos que o fato dos estudos terem sido escritos em um idioma diferente da língua nativa da autora principal deste trabalho, de alguma forma pode produzir dificuldades na tradução e compreensão de alguns aspectos que o envolvimento maior com a língua e a cultura possivelmente teria dirimido, contudo todo o esforço foi feito para minimizar isso, sobretudo a participação de outros pesquisadores nos momentos de dúvida sobre algum aspecto.

Além disso, também foi possível constatar que mesmo realizando um processo de busca minucioso, ainda são poucos os estudos que se dedicam a analisar como os pais enxergam o tratamento fisioterapêutico dos seus filhos que apresentam DM, o que deixa claro a necessidade da realização de pesquisas que abordem de forma mais frequente e aprofundada esse tema, tendo em vista que a inclusão dos pais no processo de reabilitação é peça fundamental para se alcançarresultados satisfatórios. 


\section{REFERÊNCIAS}

1. Oliveira KW, Cortez-Escalante J, Oliveira WT, Carmo GM, Henriques CM, Coelho GE, et al. Increase in reported prevalence of microcephaly in infants born to women living in areas with confirmed Zika virus transmission during the first trimester of pregnancy - Brazil, 2015. MMWR Morb Mortal Wkly Rep. 2016;65(9):242-7.

https://doi.org/10.15585/mmwr.mm6509e2

2. Reis RP. Microcephaly outbreak in Brazil. REME Rev Min Enferm. 2015;19(4):812-4. Doi: http://www.dx.doi.org/10.5935/14152762.20150061

3. Brasil. Ministério da Saúde. Secretaria de Vigilância em Saúde. Vírus Zika no Brasil: a resposta do SUS [recurso eletrônico]. Brasília: Ministério da Saúde; 2017 [Citado 2018 Set 11]. Disponivel

em: http://bvsms.saude.gov.br/bvs/publicacoes/virus_zika_brasil_res posta_sus.pdf

4. Garcia LP. Epidemia do vírus Zika e microcefalia no Brasil: emergência, evolução e enfrentamento. Rio de Janeiro: Instituto de Pesquisa Econômica Aplicada -IPEA; 2018 [Citado 2018 Set 11]. Disponível

em: http://repositorio.ipea.gov.br/bitstream/11058/8282/1/td_2368. pdf

5. Pletsch MD. Deficiência múltipla: formação de professores e processos de ensino-aprendizagem. Cad Pesqui. 2015;45(155):1229. Doi: https://doi.org/10.1590/198053142862

6. Associação Brasileira de Fisioterapia Neurofuncional. Parecer sobre estimulação precoce e microcefalia [Texto na Internet]. Rio de Janeiro: ABRAFIN; c2016 [Citado 2018 Ago 15]. Disponível em: http://abrafin.org.br/wp-content/uploads/2015/02/PARECERMICROCEFALIA.pdf

7. Lopes GB, Kato LS, Corrêa PRC. Os pais das crianças com deficiência: reflexões acerca da orientação em reabilitação motora. Psicol Teor Prat. 2002;4(2):67-72.

8. Noblit GW, Hare RD. Meta-ethnography: synthesizing qualitative studies. Newbury Park: Sage; 1988.

9. Alencar ES, Almouloud SA. A metodologia de pesquisa: metassíntese qualitativa. Reflexão e Ação. 2017;25(3):204. Doi: http://dx.doi.org/10.17058/rea.v25i3.9731

10. Lopes AL, Fracolli LA. Revisão sistemática de literatura e metassíntese qualitativa: considerações sobre sua aplicação na pesquisa em enfermagem. Texto Contexto Enferm. 2008;17(4): 771-8. Doi: https://doi.org/10.1590/S0104-07072008000400020

11. Neves RF, Nunes MO, Magalhães L. As interações entre os atores no retorno ao trabalho após afastamento por transtorno mental: uma metaetnografia. Cad Saude Publica. 2015;31(11):1-16. Doi: https://doi.org/10.1590/0102-311X00029215

12. Tong A, Sainsbury P, Craig J. Consolidated criteria for reporting qualitative research (COREQ): a 32-item checklist for interviews and focus groups. Int J Qual Health Care. 2007;19(6):349-57. Doi: https://doi.org/10.1093/intqhc/mzm042

13. Evans-Rogers DL, Sweeney JK, Holden-Huchton P, Mullens PA. Short-term, intensive neurodevelopmental treatment program experiences of parents and their children with disabilities. Pediatr Phys Ther. 2015;27(1):61-71. Doi: https://doi.org/10.1097/PEP.0000000000000110

14. Stefánsdóttir S, Egilson ST. Diverging perspectives on children's rehabilitation services: a mixed-methods study. Scand J Occup Ther. 2016;23(5):374-82.

Doi: https://doi.org/10.3109/11038128.2015.1105292

15. Kruijsen-Terpstra AJ, Verschuren $O$, Ketelaar M, Riedijk L, Gorter JW, Jongmans MJ, et al. Parents' experiences and needs regarding physical and occupational therapy for their young children with cerebral palsy. Res Dev Disabil. 2016;53-54:314-22. Doi: https://doi.org/10.1016/j.ridd.2016.02.012
16. Jindal P, MacDermid JC, Rosenbaum P, DiRezze B, Narayan A. Perspectives on rehabilitation of children with cerebral palsy: exploring a cross-cultural view of parents from India and Canada using the international classification of functioning, disability and health. Disabil Rehabil. 2018;40(23):2745-55. Doi: https://doi.org/10.1080/09638288.2017.1356383

17. Sukeri S, Bakar RS, Othman A, Ibrahim MI. Barriers to unmet needs among mothers of children with disabilities in Kelantan, Malaysia: A qualitative study. J Taibah Univ Med Sci. 2017;12(5):424-9. Doi: https://doi.org/10.1016/j.jtumed.2017.05.002

18. Lillo-Navarro C, Medina-Mirapeix F, Escolar-Reina P, MontillaHerrador J, Gomez-Arnaldos F, Oliveira-Sousa SL. Parents of children with physical disabilities perceive that characteristics of home exercise programs and physiotherapists' teaching styles influence adherence: A qualitative study. J Physiother. 2015;61(2):81-6. https://doi.org/10.1016/j.jphys.2015.02.014

19. Fitzgerald N, Ryan P, Fitzgerald A. Team-Based Approaches in Early Intervention Services for Children With Disabilities: Irish Parents' Experiences. J Policy Pract Intellect Disabil. 2015;12(3):199-209. Doi: https://doi.org/10.1111/jppi.12126

20. Nakamanya S, Siu GE, Lassman R, Seeley J, Tann CJ. Maternal experiences of caring for an infant with neurological impairment after neonatal encephalopathy in Uganda: A qualitative study. Disabil Rehabil. 2015;37(16):1470-6. Doi: https://doi.org/10.3109/09638288.2014.972582

21. Sari FL, Marcon SS. Participação da família no trabalho fisioterapêutico em crianças com paralisia cerebral. Rev Bras Crescimento Desenvolvimento Hum. 2008;18(3):229-39.

22. Larsson I, Miller M, Liljedahl K, Gard G. Physiotherapists' experiences of physiotherapy interventions in scientific physiotherapy publications focusing on interventions for children with cerebral palsy: a qualitative phenomenographic approach. BMC Pediatr. 2012;12:90. Doi: https://doi.org/10.1186/14712431-12-90

23. Gannotti ME, Christy JB, Heathcock JC, Kolobe TH. A path model for evaluating dosing parameters for children with cerebral palsy. Phys Ther. 2014;94(3): 411-21. Doi: https://doi.org/10.2522/ptj.20130022

24. Willrich A, Azevedo CCF, Fernandes JO. Desenvolvimento motor na infância: influência dos fatores de risco e programas de intervenção. Rev Neurocienc. 2009;17(1):51-6.

25. Rinaldi LM. Famílias de crianças com paralisia cerebral: subsídios para a elaboração de uma proposta de intervenção fisioterapêutica centrada na família [Dissertação]. Campinas (SP): Universidade Estadual de Campinas; 2012.

26. Uchôa AC, Vieira RM, Rocha PM, Rocha NS, Maroto RM. Trabalho em equipe no contexto da reabilitação infantil. Physis. 2012;22(1):385-400. Doi: https://doi.org/10.1590/S010373312012000100021

27. Rotta NT. Paralisia cerebral, novas perspetivas terapêuticas. J Pediatr. 2002;78:S48-S54. Doi: https://doi.org/10.1590/S002175572002000700008

28. Izumi AY. A concepção da criança com deficiência neuromotora e seus pais sobre a fisioterapia: estudo qualitativo [Dissertação]. Londrina (PR): Universidade Norte do Paraná; 2012.

29. Bernardo AC. Qualidade de vida, necessidades terapêuticas e preocupações de pais de crianças com necessidades especiais abordagem centrada na família [Dissertação]. Lisboa: Escola Superior de Tecnologia da Saúde de Lisboa; 2012.

30. Mello R, Ichisato SM, Marcon SS. Percepção da família quanto à doença e ao cuidado fisioterapêutico de pessoas com paralisia cerebral. Rev Bras Enferm. 2012;65(1):104-9. Doi: https://doi.org/10.1590/S0034-71672012000100015 
31. Lima RA. Envolvimento materno no tratamento fisioterapêutico de crianças portadoras de deficiência: compreendendo dificuldades e facilitadores [Dissertação]. Belo Horizonte (MG): Universidade Federal de Minas Gerais; 2006.

32. Barbosa AS, Santos LS, Santana AF, Monteiro LF. A participação da família no trabalho de reabilitação da criança com microcefalia. Cad Graduação. 2017;4(2):189-202.

33. Levandowski M, Carrilho L. Expectativa dos pais de crianças com patologias neurológicas em relação à fisioterapia. Rev Saúde Integrada. 2012;107-31.

34. Pedroso CN, Félix MA. Percepção dos pais diante do diagnóstico e da abordagem fisioterapêutica de crianças com paralisia cerebral. Ciência \& Saúde. 2014; 7(2):61-70. Doi: https://doi.org/10.15448/1983-652X.2014.2.16464

35. Souza SC, Pires PA. Comportamento materno em situação de risco: mães de crianças com paralisia cerebral. Psicol Saúde Doenças. 2003;4(1):111-30.
36. Cerqueira MM, Alves RO, Aguiar MG. Experiências vividas por mães de crianças com deficiência intelectual nos itinerários terapêuticos. Ciênc Saude Coletiva. 2016;21(10):3223-32. Doi: https://doi.org/10.1590/1413-812320152110.17242016

37. Baltor MR, Dupas G. Experiências de famílias de crianças com paralisia cerebral em contexto de vulnerabilidade social Método. Rev Latinoam Enferm. 2013; 21(4):956-63. Doi: https://doi.org/10.1590/S0104-11692013000400018

38. Araújo YB, Collet N, Moura FM, da Nóbrega RD. Conhecimento da família acerca da condição crônica na infância. Texto Context Enferm. 2009;18(3):498-505. Doi: https://doi.org/10.1590/S0104-07072009000300013

39. Gração DC, Santos MG. A percepção materna sobre a paralisia cerebral no cenário da orientação familiar. Fisioter Mov. 2008;21(2):107-13. 